\title{
THE NEXT DECADE OF THEORETICAL SOLAR PHYSICS
}

\author{
M. KUPERUS \\ Astronomical Institute, University of Utrecht, The Netherlands
}

\section{Introduction}

Exactly a decade ago an international conference on solar physics took place in Utrecht on the occasion of the late Prof. Minnaert's 70th birthday. Since then, much has happened. Many new instrumental devices were developed or refined. Completely new observational techniques were applied in the XUV and radio wavelengths and in particle detection.

This all resulted in an explosion of new data. Moreover new problems appeared of course. It also resulted in an explosion of papers on solar physics. Actually many more contributions appear than reasonably can be absorbed by the periodical Solar Physics, of which the first volume appeared in 1967. At that time the editors were not at all sure that they would receive enough papers to maintain such a journal. But were there major problems solved recently?

At first I would be inclined to think that, notwithstanding the enormous efforts spent in solar physics, little has been achieved and no spectacular breakthroughs have been found. This impression, probably shared by many more colleagues, becomes stronger if one tries to find a line of progress in 'classical' problems of solar physics. We still do not have a satisfactory theory of the Sun's differential rotation and the Sun's outer convection zone. One may argue that this is due to our bad knowledge of the interior structure which is not accessible to direct observation. Solar activity and the formation of sunspots is now believed to be a direct consequence of differential rotation and cyclonic convective motions and therefore suffers from the same uncertainties.

But also in the outer observable layers we have still a long way to go. We do believe that the photospheric motions and the very high temperature of the corona are causally related, but only occasionally a piece of evidence is found that fits the complicated puzzle of the heating of the corona.

The greatest mystery on the Sun is the solar flare. Though known for decades it seems that we have not come further than a few controversial theories embedded in thousands of observations of very different and sometimes contradictory character. We only poorly understand how high energy particles may be generated and an understanding of the various plasma processes responsible for the sporadic radio radiation is just beginning.

This may all sound discouraging but it rather shows how complicated solar physics is at this moment. In Minnaert's talk on solar physics in 1963 he reviewed a period that terminated an era of great discoveries in solar physics: Magnetic fields could be measured on the sun and it had been found that the corona is a very hot plasma. 
It was in that period that it became clear that the solar corona was expanding with supersonic velocities: the solar wind. The oscillatory motions were discovered which are in fact the non-thermal energy source which produces the corona and the solar wind. Nowadays we have to do a much harder job to proceed. The pioneers have roughly explored the Sun and an army of settlers is trying to make a living of it.

What is it then that we gained in the last ten years? I would like to make this clear by the following comparison. In 1950 one could describe the Sun as follows:

'The Sun is a thermally radiating gaseous sphere'.

In 1970 I would describe the sun in a more complicated manner:

"The Sun is a rotating magnetic plasma with a complex transient XUV, optical, radio and cosmic ray spectrum"

Around 1950 one was able to construct a spherical model of the solar atmosphere once the source of the continuous opacity was understood. However the abundances could already be determined from the relatively 'simple' optical spectrum. And thus a self consistent picture of the solar atmosphere could be developed. The atmospheric model thus determined was basic in the sense that one could now try to calculate line profiles and use these as diagnostic tools. However, here the first problems already appeared since the Sun is not spherically symmetric on a small scale and certainly in the outer layers the plasma properties are more pronounced than the gaseous properties.

Hence this is present day's situation. A theoretical solar physicist has to study plasmaphysics, magnetohydrodynamics, gasdynamics, non-LTE physics, high-energy physics and general relativity. A non-evolved 1950 solar physicist would feel as ignorant as a schoolboy. This situation can be found in almost all modern branches of astrophysics but it is exceedingly clear in solar physics.

This should elucidate that progress in solar physics should be seen in the context of a mature sophisticated science. There are astronomers for whom the Sun is nothing else than an ordinary main-sequence G2 star. I would classify those people among the non-evolved 1950 astronomers, who do not realize how uninteresting a star or a galaxy is if you are too far away to get the proper observational information.

The next decade in theoretical solar physics will probably be characterized by the understanding that the Sun is the nearest cosmical laboratory in which a large class of astrophysical problems can be studied in detail. A great number of physical processes that are held responsible for phenomena in distant objects occur in the Sun. A study of these processes is absolutely necessary for the understanding of the universe and is thus of general astrophysical importance.

What are these problems? I want to make a personal selection in the hope that others will fill in the gap I leave.

For example I will deliberately omit spectroscopy and line formation. Firstly because I am not well aware of the latest developments in this field but secondly because I do not think it belongs to what I consider theoretical solar physics should be. Spectroscopy should be considered as a general astrophysical technique. It is part of a measuring technique. In fact it is a sophisticated thermometer, barometer, kinometer, voltmeter or magnetometer. This important branch of solar physics belongs to applied- 
solar physics. Spectroscopy supplies the crucial data after reduction of the observations.

In theoretical (solar) physics the idea should be placed central and should be based on the fundamental laws of physics. Single observations may be of little value as long as there is no idea about the principle physical processes that may take place. At this point I may quote Einstein (Mein Weltbild):

"Solange die Prinzipe, die der Deduktion als Basis dienen können, nicht gefunden sind, nützt dem Theoretiker die einzelne Erfahrungstatsache zunächst nichts, ja, er vermag dann nicht einmal mit einzelnen empirisch ermittelten allgemeineren Gesetzmässigkeiten etwas anzufangen."*

I will briefly review the present situation and the expected developments in the subsequent topics which I consider most important for theoretical solar physics.

- Internal structure

1. neutrino problem

2. oblateness

3. rotation and convection.

- Structure of the photosphere and the chromosphere

1. photospheric structure, granulation, sunspots

2. chromospheric structure, spicules

3. waves in the atmosphere.

\section{- Corona}

1. transition region between chromosphere and corona

2. heating of the corona

3. solar wind

4. coronal structure

5. coronal instabilities and non linear plasma phenomena.

- Solar magnetic fields

1. solar dynamo

2. small scale magnetic fields

3. solar flares and prominences.

\section{Internal Structure}

\subsection{NeUTRINO PROBLEM}

One of the most mysterious problems in solar physics is the so called 'Case of the Missing Neutrinos'. Neutrinos are supposedly generated in the solar interior as resulting products of the proton cycle. The proton cycle has several branches. One of them has two major neutrino producing reactions.

* As long as the principles, which can serve as a basis for the deduction, have not been found, single empirical facts are at first of no use to a theoretician, in that case he even does not know what to do with single empirically obtained general relations. 
The neutrinos produced by the first reaction $p+\bar{e}+p \rightarrow v$ are called the 'PEP' neutrinos. The neutrinos produced by the second reaction $\mathrm{B}_{5}^{8}\left(e^{+} v\right) \mathrm{Be}_{4}^{8}$ are called the Boron neutrinos. These neutrinos have different energies. The experiment by Davis using the detection reaction ${ }_{17}^{37} \mathrm{Cl}\left(v, e^{-}\right){ }_{18}^{37} \mathrm{~A}$, is able to detect the Boron neutrinos.

It was found that the solar neutrino flux is a factor 10 smaller than predicted by the theory. It is a great step forward that the solar neutrinos have been detected now with a beautiful experiment, but it appears to be one of the mysterious problems in astronomy that there are neutrinos missing.

Several suggestions have been made to solve this enigma.

(a) $\mathbf{B}^{8}$ is not formed at all in the Sun, but up to now one does not know of a destructive reaction.

(b) The solar interior is not stationary burning but shows a periodic or quasiperiodic kind of activity. Due to the long transfer time for the radiation, the observed solar radiative flux could then be related to some activity in the past, while the neutrino flux reflects the present internal structure.

(c) The neutrinos have already partly decayed before they reach the Earth.

(d) Models with a fast differentially rotating core, show a decrease in neutrino production. However to reconcile a model with the neutrino observations, the oblateness should be larger than observed.

Many more suggestions will be made in the near future. It is a fascinating problem that may give us a direct understanding and check of the nuclear processes in stellar interiors.

\subsection{SOLAR OBLATENESS}

A second problem that has drawn a lot of attention is the magnitude of the solar oblateness. It started with the experiment by Dicke and Goldenberg in 1966 which demonstrates that the oblateness $\Delta r / r=5 \times 10^{-5}$ instead of the expected value of $10^{-5}$, which would be in agreement with the rotation period of 27 days. An oblateness of $5 \times 10^{-5}$ means that there is a difference of $35 \mathrm{~km}$ between the equatorial and the polar radius. The important thing is that this oblateness would explain the perihelion motion of Mercury of 4 / /century within the framework of the scalar-tensor theory of Brans and Dicke.

The experiment looked very clear and convincing since the measurements seem to show a seasonal variation, which is supposedly due to the variation in the position of the solar rotation axis.

The conclusion must therefore be that the Sun possesses a rapidly rotating inner core.

Several theoretical arguments have been put forward against a rapidly rotating core:

(a) There would be a spin down due to the formation of a viscous Ekman layer. However, we do not yet know sufficiently well what the precise influence of the density gradient is.

(b) The Goldreich, Schubert, Fricke instability would prevent a core from fast rotation. However, if sufficient angular momentum is transported outward through 
mass loss, the envelope could be braked while the core keeps spinning rapidly.

(c) The observations could be explained by assuming a difference in temperature of $40 \mathrm{~K}$ at $\tau_{0}<0.05$. A correlation with faculae has been made. This may explain about $30 \%$ of the measured value.

It has recently been shown from a direct measurement of $\Delta T$ and a correlation with magnetic fields that a pole-equator temperature difference is not sufficient to explain Dicke's measurements. What is needed is a refinement of measurements of perihelion movements of other planets. Finally a refined analysis of the as yet not published measurements is needed. A repetition of the experiment in some form is certainly useful.

\subsection{SOLAR ROTATION AND CONVECTION}

The rotation of the Sun and especially the differential rotation is an old and still not well understood problem. What is the influence of the convective envelope on the rotation? Are there meridional circulations? Is differential rotation a superficial phenomenon or does it reflect the non-uniform rotation of the Sun's interior layers?

Due to the interaction of convection with the rotation a difference in flux between the pole and the equator can develop inside the convection zone. These differences may become very small in the observable layers due to large-scale meridional circulation. Due to these circulations a differential rotation in the upper part of the convection zone may be generated. The upwelling occurs at the poles and the sinking at the equatorial latitudes. This would however suggest that the polar regions are convectively more unstable than the equatorial regions which is just opposite to the stabilizing influence of the rotation at the poles.

A suggestion that has been made and that will be interesting to work out is that deep inside the convection zone the circulation occurs from a slightly hotter equator towards a slightly cooler pole. A counter cell would then account for the right direction of circulation.

The meridional circulation of course transports angular momentum which could be balanced in a steady state by viscous transport in the direction antiparallel to a gradient in angular velocity.

It is clear from the above discussion that the missing link in any theory of the differential rotation is the absence of a good theory of turbulent convection, or even a good approximation to it.

A different approach is that differential rotation is maintained not by meridional circulation but through momentum transport in the convective eddies.

Calculations, however, show that the meridional currents are in that case directed towards the poles.

A major problem to be solved is compressible non-boussinesq convection and an answer should be given to the question: How deep is the convection zone and what is the effect of coriolis forces on the heat transport and thus in turn on the circulation and on the differential rotation. 


\section{Structure of the Photosphere and the Chromosphere}

\subsection{Photospheric Structure, Granulation, SUnspots}

The study of the photosphere and chromosphere is the oldest in solar physics. Yet there are a few important unsolved problems.

Is the solar granulation a pure convective mode or does it have an oscillatory character? More high resolution measurements seem needed at this time. The fine structure of the photosphere can give us valuable information about the state of motion and the modes of energy transfer in the subphotospheric layers. Moreover they are an indispensable piece of information for any theory of the structure and heating of the overlying atmospheric layers.

It seems that there are at this moment no outstanding theoretical problems in the physics of the quiet photosphere. However it would be very helpful for the structure of the overlying layers to know the wavenumber spectrum and the frequency spectrum of the photospheric turbulence and the granulation. This would yield the output of mechanical energy that is supposed to maintain the corona.

Again small scale photospheric magnetic fields such as the recently observed subgranular filigree structure are certainly of importance, not in the first place to clarify the photospheric properties, but more to serve as a set of lower boundary conditions, which are necessary to understand the upper chromosphere and the corona (see Section 5.2).

Inhomogeneous models of the quiet photosphere and the faculae should only be constructed if one knows the type of inhomogeneity. It is not very useful to refine solar atmospheric models which depend on too many parameters, which are at this moment unknown. Only models that are physically consistent should be used.

A major problem in theoretical photospheric and subphotospheric physics is the nature of sunspots. Our knowledge about the origin of these magnetic markers is very meagre. The formation of sunspots can not be considered apart from the dynamo mechanism of solar magnetic fields, which will be discussed presently (Sections 5.1 and 5.2) Several empirical models have been constructed which are all not fully consistent. The radiation field in the low chromospheric layers of the sunspot is very complex due to lateral deviations in the temperature. It is important to study sunspot problems after the observations have been reduced with a proper theory of line formation applicable to these circumstances. Many peculiarities that are frequently reported may then appear of no value.

Oscillatory motions inside sunspots have been reported. They may be very useful as additional diagnostic aids where the definition of physical properties becomes ambiguous. Oscillations and Evershed flow are probably linked with the energy transport in umbrae and penumbrae.

There is at present no satisfactory theory for the formation and decay of sunspots. What is needed is a complete magnetohydrodynamic analysis of the convection and circulation in the outer convective layers when reasonably strong magnetic fields are present. The exact place where a pore or small magnetic spot occurs in the super- 
granulation pattern could yield valuable information. As for the photosphere in general, also here any static homogeneous model should be regarded with doubt from a theoretical point of view.

The decay of sunspots occurs too rapidly if one considers Ohmic diffusion as the major process. There are several ways out of this problem. Firstly, the current system is present in thin shells which then diffuse much faster than the whole spot due to the large gradients at the edge. Secondly, the electrical conductivity in the turbulent solar plasma is effectively much smaller than the usual Coulomb conductivity. Observations of the associated velocity fields just around the sunspot may clarify this. In particular the outflow of magnetic flux as is recently observed, is more an indication for a gradual destruction of the sunspot by removal of flux then for the Ohmic dissipation.

The problem of sunspot formation should be studied together with the more general problem of the birth, growth and decay of solar active regions and solar faculae. We will return to this when discussing solar magnetic fields (Sections 5.1 and 5.2).

\subsection{Chromospheric STRuCTURE, SPICULES}

Recently chromospheric structure is paid a great amount of attention since high resolution $\mathrm{H} \alpha$ filters and XUV spectroheliographs became available. It may well happen that within a few years not many theoreticians will be interested any more in the current problems of chromospheric structure and chromospheric dynamics, because high resolution extraterrestrial observations in a wide range of wavelengths (Skylab, Spacelab etc.) will probably solve these problems.

A great number of ideas has been developed in the last years and a lot of thinking is going on. It is now the observer who should decide which of the various ideas he considers consistent with the observations.

But it is not yet so far, we are still puzzled by the very nature of the chromospheric spicules, which seem to be a physical connection between the chromosphere and the corona, not only positionally but especially as a mode of energy transfer.

At first it seems logical to consider the spicules, driven by the momentum of the chromospheric waves as a kind of visualisation of the shock waves which are required to heat the corona.

But spicules may as well be produced by an energy inbalance in the upper chromospheric layers. In that case they are thermally generated instead of momentum generated. Even radiation pressure due to absorption in strong spectral lines such as $\mathrm{L} \alpha$ is considered as a possible cause for chromospheric instability.

It will be necessary again to decide from the observations which of these ideas can be applied. Magnetic field measurements in the chromosphere or at least good magnetically correlated data would be very welcome.

\subsection{WAVES IN THE ATMOSPHERE}

A quantity of great uncertainty is the flux of non-thermal energy in the photosphere, the chromosphere and the corona. 
The only definite evidence for a flux of mechanical energy is the 5-min oscillatory motion observed in the photosphere and the chromosphere. However the nature of these oscillations is still obscure. It is not yet clear whether they are acoustic or gravity waves or of the evanescent type. In the case the waves are generated by the convective boundary or the granulation elements they are probably gravity waves or magnetically modified gravity waves in regions with enhanced magnetic field.

Waves which are generated subphotospherically in the turbulent convective layers are acoustic waves. These waves may be trapped in the photosphere-chromosphere temperature trough to cause standing oscillations. For waves which are generated in the turbulent convective layers a very strong directional dependence occurs. Much more energy is transmitted in the direction of the mean convective velocity than perpendicular to it. This would result in a very inhomogeneous distribution of wave sources over the solar surface.

Another problem which is not yet solved is the effect of the radiative decay of low frequency waves. The radiative diffusion time is so short in the low photospheric layers that it is hard to maintain 5-min oscillations so low down. This could be used as an argument that these oscillations are actually generated in the higher chromospheric layers where the radiative relaxation time is much longer.

It is possible that the waves are acoustic waves which can easily pass the temperature minimum but are modulated by low frequency oscillations. The carrier waves are then not influenced so much by radiative relaxation.

In medium strong magnetic fields the Alfvén waves must play an important role in the net non-thermal energy transport. When generated in the upper chromosphere they are mainly undamped up to large distances in the corona but in strong magnetic fields they can propagate from the lower chromosphere into the corona. Mode coupling between gravity waves and Alfvén waves enable Alfvén waves to be generated and to propagate upward without frequency limitations.

In a medium with a constant temperature and a constant magnetic field strength the amplitude of Alfvén waves depends on the density as $v \sim \varrho^{-1 / 4}$ while the amplitude of acoustic waves depends on the density as $v \sim \varrho^{-1 / 2}$. Therefore non-linear steepening is more pronounced for the acoustic waves. They will become shock waves already low in the atmosphere. In this sense there are two direct lines of coupling.

First: from convection either via gravity waves or directly into Alfvén waves.

Second: from turbulence via acoustic waves into shock waves.

Many interrelations are possible, most of them are not yet well studied.

a. corona

b. chromosphere

c. photosphere

c. convection zone

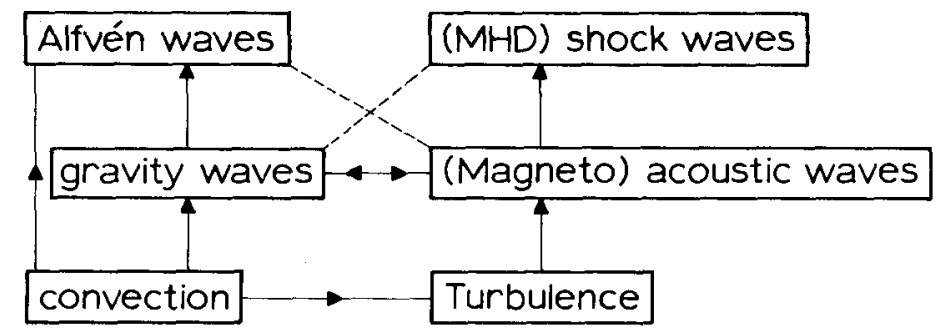


Finally a careful search should be made on the interesting possibility for the thermal generation of waves. Especially in the upper chromospheric layers an energy inbalance may result in motions which predominantly travel upwards and may form shock waves. They may well be the source of the spicules as has been suggested before.

But also hydromagnetic waves may be generated in thermally unstable layers traversed by a magnetic field.

It is worthwhile to investigate whether the kappa-mechanism, well known from the theory of Cepheid and RR-Lyrae pulsations may be operative in the solar ionization zones and thus may drive the photospheric and chromospheric oscillations.

\section{The Corona}

\subsection{THE TRANSITION REGION BETWEEN CHROMOSPHERE AND CORONA}

It has long been recognized that the transition from the chromosphere to the corona occurs very abruptly with an extremely large temperature gradient $(\mathrm{d} T / \mathrm{d} h \approx$ $\approx 10^{2}-10^{3} \mathrm{~K} \mathrm{~cm}^{-1}$ ) and that a large part of the heat dumped into the corona is conducted backwards into the chromosphere, due to the very large coefficient of thermal conduction $\left(\varkappa \sim T^{5 / 2}\right)$.

I think it is one of the great achievements in solar physics of the last decade that the very sharp rise in temperature in the transition region has been observationally established, thanks to the interpretation of the XUV observations (OSO-satellites). The method which is used to determine the temperature structure of the transition region from the XUV line intensities also yields the abundance of several elements.

Since the transition region is largely conduction dominated and because the coefficient of thermal conduction is strongly reduced perpendicular to the magnetic field, thereby causing a strong channelling of the heat flow along the magnetic field lines, the transition layer will follow closely the inhomogeneous structure in the magnetic field. The temperature distribution in the supergranular boundaries, where the field is concentrated, is therefore different from the temperature distribution at the region in between the magnetic concentrations.

At this moment it is hard to say whether the temperature gradient above the magnetic mottles is smaller or greater than in between. It depends on many other parameters such as the mechanical energy flux in both regions, which in turn determines the conductive flux. The value of the conductive flux which is frequently found in the literature is $F_{c}=3-6 \times 10^{5} \mathrm{erg} \mathrm{cm}^{-2} \mathrm{~s}^{-1}$. If the XUV observations are interpreted taking reasonable inhomogeneous structure into account, this value may be an order of magnitude lower. High resolution XUV observations must solve this problem.

The thermal stability and the dynamical stability of such a thin layer where the temperature rises two orders of magnitude remains an open question. Is the radiative output of the upper chromosphere able to dispose of the downward conductive energy flux? How does the transition layer respond to fluctuations in energy input? Are the spicules required to match the energy balance? Is the transition region the breeding ground of all kinds of thermally driven motions which may well propagate high into 
the corona. How does the transition region respond to a disturbance from below such as a shock wave. These questions should be investigated taking into consideration the magnetic field structure, which will certainly dominate the dynamics of the transition region.

A problem of particular interest is the mass flux through the transition region. Spicules seem to provide almost hundred times more mass flux than is needed to supply the quiet day solar wind. This means that most of the mass ejected by spicules in some way must return to the chromosphere. Have these downward motions escaped observation or are there almost as many spicules with downward motions as with upward motions.

\subsection{Heating OF THE CORONA}

It is generally accepted that the corona owes its existence to the dissipation of mechanical energy transported by low frequency waves of acoustic nature from the photospheric layers into the corona.

Nevertheless a detailed theory is lacking which is not surprising if one takes into account on how many detailed physical processes such a theory depends. Moreover there are really no pertinent observations that may give conclusive evidence as to one of these many physical processes, so that one has to rely mainly on theoretical predictions. This situation is likely to remain unchanged for some years. Unfortunately the density structure, which is the easiest to determine, gives very little information on the heating mechanism. What is needed is a detailed knowledge of the temperature structure parallel as well as perpendicular to the magnetic field. One ought to know the proton temperature as well as the electron temperature not only in the outer corona but also at the 'base' of the corona.

In the upper chromosphere and the lower corona the dissipation is very likely provided by ordinary viscosity and thermal conduction in non-linear acoustic or magnetoacoustic (shock) waves. Alfvén waves probably do not contribute to the heating of the chromosphere because they may travel almost undamped into the corona.

Already in the lower corona but certainly in the upper corona the plasma properties are such that even for the low frequency waves the plasma may be considered as collisionless. In that case proton Landau damping of ion acoustic waves (which is the equivalent of an acoustic wave in a neutral gas) is the main energy source for the heating of the corona. Electron heating by direct Landau damping may be efficient in the upper layers of the corona. Such waves are not likely to be the direct remnants of waves, which would have been generated in the photosphere or below. They are probably of local origin generated as a consequence of the interaction of other wave modes or of the instability of plasma streams along the magnetic field.

Besides a study of wave damping, which is wave-particle interaction, in collisionless plasma's, the theory of the heating of the corona can only proceed if a profound study is made of the various possibilities of mode coupling in (collisionless) plasma's.

An interesting possibility to obtain some information on wave propagation in the or ona is from the study of fine structure in high resolution radiospectra. There are a 
number of non-thermal long-lived phenomena associated with an active region like type I storms, $D_{m}$ type III storms, long lived fast particle streams, all not flare related, whose understanding would greatly benefit from a study of the collisionless processes during the propagation of magnetohydrodynamic waves in the corona.

\subsection{THE SOLAR WIND}

There has been a rapid progress in the general knowledge of the solar wind and the structure of the interplanetary medium, mainly from the observational point of view, essentially because of the developments in space research. However, there remain many theoretical problems to be solved since the energy sources are just as badly known as for the heating of the corona.

Collective interactions are fundamental in the interplay between systematic flow, magnetic field, rotation and different particle species, with different distribution functions in various directions with respect to the magnetic field. Evidence for the existence of plasma turbulence in the solar wind is found in the radio scattering data, radio observations of long lived sources and fast particle stream detection.

The study of the excitation, propagation and decay of different wave modes and their influence on the flow-velocity and the particle distribution function is of prime importance for the understanding of the solar wind. It might however be unrewarding to spend too much effort in trying to find the complete distribution function and the electromagnetic fluctuation spectrum in space and time. More physical insight would be gained from the study of the effect on the solar wind from evolutionary and sudden changes of boundary conditions thereby using simplified models. Such studies are necessary to understand e.g. the interplanetary sectors and the evolution and interaction of streamers.

It should be noted here that these problems are not only of interest for solar physics. Hot dilute rotating magnetoplasma occurs near other stars, in $\mathbf{H}^{+}$regions, supernovae, pulsars, galactic nuclei and quasars. The solar corona offers a first hand opportunity to test the physical theories applicable to such a plasma, using particle, $\mathrm{X}$-ray and radio data.

\subsection{Coronal STRUCTURE}

The lower corona, strongly influenced by inhomogeneous photospheric magnetic fields, exhibits a wide variety of fine structure, which is partially reflected in the inhomogeneous and variable radio radiation. Considerable work has been done on the structure of coronal streamers and I feel that the study of the physics of neutral sheets is of prime importance. The detailed understanding of the plasma physical processes in a neutral sheet is still lacking. Anomalous conductivity, wave-wave, wave-particle processes, wave and particle propagation inside the streamer, the influence of parallel flow on the stability can be handled by the physics known today. As said before, the temporal evolution of inhomogeneous structures is still in a first stage, but especially the birth and decay of a streamer, with and without helmet structure should be investigated. 
As was to be expected, the average coronal structure is related to large photospheric regions with weak average magnetic field, and much less to small regions with large field strengths. The exact connection however, the development in time depending on the photospheric boundary conditions, is still quite unknown.

Besides streamers we encounter coronal holes, plasma nodules, filaments, coronal condensations. Interesting tests for the theory are provided if these features are disturbed by flaie driven shocks or particle injections.

\subsection{CoRONAL INSTABILITIES AND NON-LINEAR PHENOMENA}

Current theories of type III bursts indicate that the growth of plasma waves by a beam-plasma instability has a much smaller time scale than the duration of the beam, and the actual observed profile is more determined by the non-linear processes that stabilize a particle beam pervading the plasma, than by the linear growth and decay of the beam-plasma instability.

It thus appears necessary to calculate non linear coupling coefficients in order to describe the stabilized situation.

A good qualitative study of a particle beam inside or outside a coronal streamer and a comparison with the observations seems possible now. This may well clarify the problem of the occurrence of harmonic-fundamental pairs and fine structure and also to what extent type III bursts can be used as coronal probes revealing the coronal structure.

Above active regions, up to large heights in the corona, a high level of low frequency plasma turbulence is to be expected, which can generate fast particles which in turn can produce turbulence with higher frequency. The effects will be quite different for regions with open radial field lines than for those with closed field lines. In the latter case the energy transport can be blocked at a certain height leading to an explosive situation, while in the case of open field lines the heat flow is unimpeded and a coronal hole may arise.

Although type III bursts seem to offer a well-defined problem, wuch less is known about stationary type IV bursts with their detailed spectral fine structure. An interesting starting point is to study the relaxation of a hot plasma cloud trapped in a closed magnetic field structure. This is also of importance for understanding the magnetosphere and its interaction with the solar wind.

\section{Solar Magnetic Fields}

\subsection{THE SOLAR DYNAMO}

The last decade of theoretical solar physics has brought a great understanding of the nature of the 22-yr cycle of solar activity. It is generally accepted that the solar dynamo results from two types of motions: differential rotation and cyclonic convection or/and turbulence.

The basis of any dynamo theory is as follows: At solar minimum the field is essentially poloidal. Differential rotation generates a strong toroidal field which becomes 
visible (at the surface) first at medium latitudes and then at lower latitudes. The polarity laws can be explained in this way. The toroidal field emerges in active regions of bipolar nature. During the emergence of the toroidal field either by convective motions or through kink instability the field received a poloidal component of opposite polarity as the initial poloidal field during minimum activity. Supergranular motions then spread the fields over the solar surface and a new poloidal field of reversed sign is formed. Then the same processes are repeated with reversed signs to complete the magnetic 22-yr cycle.

It is known that dynamo's must be non-axisymmetric. Cyclonic convection caused by the Coriolis force and helical turbulence (turbulence for which $\langle\mathbf{u} \cdot \operatorname{curl} \mathbf{u}\rangle \neq 0$ ) have this property and are most likely to play an essential role in the formation of a poloidal field out of toroidal fields.

The effect of helical turbulence in a magnetic field is to generate a non curlfree electric field in the direction of the magnetic field. This mechanism, which is called the $\alpha$-effect, generates a magnetic field perpendicular to the initial magnetic field. Turbulence is very powerful in the upper layers of the convection zone. Hence the $\alpha$-effect can only be efficient in a shallow layer.

Models have been presented using the differential rotation in the deeper layers to build up toroidal fields and the $\alpha$-effect to build up the poloidal fields in the upper layers. It is found that the distance between those layers regulates the period of the cycle. These dynamo's are called $\alpha-\omega$ dynamo's contrary to the $\alpha \alpha$ dynamo's which only use the $\alpha$-effect to obtain a cycle. Thus in $\alpha \alpha$ dynamo's the toroidal fields are produced through the helical turbulence from the poloidal fields. Satisfactory butterfly diagrams have been constructed.

However it is still not clear whether the solar dynamo is a mechanism that operates on superficial magnetic fields or whether deep seated fields are involved.

Non linear calculations confirm the observed fact that the period decreases slightly with increasing field amplitude. Moreover they show the asymmetry between rising and declining branch of a cycle.

\subsection{SMall SCALE MAGNETiC FIELDS}

Once the toroidal magnetic fields have emerged through the convection zone they are spread over the whole solar surface in a random way. It has become clear that the magnetic field is not diffusively moving over the solar surface but it remains concentrated in magnetic ropes. The convective motions drag the field to the edges of the cells where they are concentrated especially in the corners of the supergranulation. This leads to fields of the order of $100 \mathrm{G}$.

It is a challenging question how fields can be amplified to values of the order of $3000 \mathrm{G}$ as observed in sunspots. No satisfactory theory of sunspot formation exists at this moment but it seems that the modification of the energy transfer in rather strong fields must play a significant role on the further amplification of these fields. As soon as convection is reasonably suppressed, the atmospheric structure of the overlying layers is changed in such a way that lateral pressure equilibrium can only be 
achieved by the compression of the magnetic field. The Evershed motions are probably a direct consequence of this process.

The subphotospheric toroidal field strings naturally emerge as bipolar magnetic regions. Some of the field tubes are already heavily twisted when they appear at the surface. It is clear that these fields contain magnetic energy that can be released in the upper atmosphere. Other field tubes may appear less violently. When they merge into the corona the solar wind will drag the weaker fields outward thus forming streamers in which the field direction changes its sign. The neutral line separating the photospheric polarities thus marks the basis of a neutral sheet. The stronger fields can resist the solar wind drag and consequently they remain present as closed loops clearly visible in he lower parts of helmet streamers.

The way in which the small scale fields emerge and the interaction of the solar wind with the coronal fields eventually determines the structure and shape of the solar corona (Section 4.4).

Although it looks as if the corona is a collection of streamers it should be stressed here that in the absence of any kind of solar activity the quiet solar corona exists which is clearly demonstrated by the 1954 eclips photograph.

\subsection{Solar flares AND PROMineNCE}

Solar flares are the most complex phenomena on the Sun. Any theory of solar flares ought to explain a great multitude of observations of enhanced radiation in the whole spectral region which takes place in a very short time. The impulsive phase lasts about $2 \mathrm{~min}$, the eruptive phase $15 \mathrm{~min}$ and after about one hour the flare eruption is over. On a very short time scale there are type III bursts lasting about $1 \mathrm{~s}$ and X-ray and microwave bursts with a duration of $0.5-2 \mathrm{~min}$.

The total amount of energy released is about $2 \times 10^{32}$ erg which is supposedly extracted from a volume of $10^{28} \mathrm{~cm}^{3}$. Only a strong magnetic field of the order of $100 \mathrm{G}$ would be sufficient if all its energy could be converted in the electromagnetic and corpuscular radiation.

There are two basic ideas which in some variation always appear in flare theories. Firstly, the X type neutral point or neutral line theory and secondly the currentdischarge theory. In the first category of theories the magnetic energy is liberated at the neutral line while in the second type of theory the magnetic energy of a current circuit is liberated at an interruption point. The last theory has the advantage that very large electric fields are created which could accelerate particles up to high energies, but it seems on the other hand very unlikely that coronal currents can be built up to such large values that an interruption occurs. Long before this could happen, the currents become kink unstable and a new configuration appears in which actually a twisted 'neutral line' is present along which the flare eruption can take place. This means that the X-type neutral line theory in a modified geometrical configuration is probably the essential core of the flare model.

But there are many questions to be solved. Which instabilities can occur? How is the plasma heated and where and when precisely are the particles accelerated? However, 
the basic problem in flare theory remains the storage of energy in suitable magnetic field configurations and the sudden release of this energy after some time.

Prominences are divided in two classes: the quiescent and the active prominences. An element common in all recent prominence theories is a flux tube which is supported against gravity either by its own tension or by ambient magnetic fields.

It can be shown that these flux tubes can originate in coronal neutral sheets during the cooling of coronal matter. Neutral sheets where fields of opposite polarity meet are the places where quiescent prominences naturally occur. There is strong evidence that quiescent prominences are a transition phase during the reconnection of the antiparallel fields.

Since over large parts of the solar surface the field is of one polarity an emerging bipolar magnetic region of much smaller extension than the surrounding unipolar region always somewhere creates a region of oppositely directed magnetic fields. They will thus occur at the border of magnetic regions, preferentially when new born magnetic fields interact with old existing fields.

The evolution and annihilation of magnetic fields which is observable in the form of flares and prominences should have a great priority in the next decade of theoretical solar physics.

\section{Conclusion}

Solar physics is a living science and one of the most sophisticated of all astronomical sciences. Many physical processes that take place in other cosmical objects but are difficult to observe can be studied in detail on the Sun.

Theoretical solar physics should lead to:

(a) an understanding of observed solar phenomena.

(b) the possibility of extrapolating this knowledge to other stars.

(c) as far as unstable plasma processes are concerned it should be investigated whether results obtained in solar physics can be scaled such as to make them applicable to galactic and extragalactic circumstances.

The subsequent list contains what I would call the major physical problems in solar physics:

I. Rotation and circulation

1. differential rotation

2. compressible non-Boussinesq turbulent convection

II. Non-thermal energy transfer

1. generation and propagation of atmospheric waves

2. heating of the corona

3. solar wind

III. Generation and evolution of magnetic fields

1. solar dynamo 
2. evolution of magnetic fields in the atmosphere e.g. sunspots

3. magnetohydrodynamics of neutral sheets, prominences

IV. Plasma instabilities

1. the mechanism of solar flares

2. plasma turbulence in the solar corona

3. acceleration of particles

I am well aware of the very personal view that is expressed in the above table. But it is still too much extended if it comes to priorities.

If I had to make a selection of what I would think are the important topics in the next decade I would with some hesitation give the highest priority to the following subjects:

- differential rotation

- heating of the corona

- birth and decay of sunspots

- magnetohydrodynamics of neutral sheets

- mechanism of solar flares

- acceleration of particles.

I hope this survey on solar physics may help to concentrate our work around the above mentioned topics.

\section{Acknowledgements}

The author thanks Drs Van Bueren, Raadu, Rosenberg, Rutten and Zwaan for many useful information and critical discussions. He is especially grateful for the many interesting discussions he had with Dr Rosenberg on this subject and for his kindness to present this paper at the IAU, General Assembly in Sydney. 